\title{
Análisis de palabras clave en la comunicación para el desarrollo y el cambio social: el caso de \#comunicambio en Twitter ${ }^{1}$
}

\author{
Keyword analysis in communication for development \\ and social change: the case of \#comunicambio on Twitter
}

\author{
MARTA SÁNCHEZ-SAUS LASERNA
}

UNIVERSIDAD DE CÁDIZ

Artículo recibido el / Article received: 2017-03-12

Artículo aceptado el / Article accepted: 2018-02-12

RESUMEN: La comunicación para el desarrollo y el cambio social (CDCS) ha experimentado en los últimos años un incremento de su protagonismo en el campo de la comunicación. No obstante, carecemos de los indicadores que nos permitan identificar objetivamente la presencia de la CDCS en la investigación sobre comunicación en España. Ante esta situación, este artículo persigue dos objetivos. Por un lado, trata de determinar los marcadores temáticos que caracterizan los discursos en Twitter sobre CDCS y, por otro lado, trata de descubrir las diferencias temáticas entre estos discursos y el discurso académico sobre CDCS en España. Para lograr estos objetivos, hemos realizado un análisis de contenido a partir de palabras clave sobre un corpus de tuits emitidos en torno al hashtag \#comunicambio y, a continuación, hemos comparado estos resultados con las palabras clave de un corpus de artículos científicos sobre CDCS publicados en las principales revistas de comunicación españolas. Los

1. La investigación que presentamos se ha elaborado en el marco del proyecto de $\mathrm{I}+\mathrm{D}$ «Evaluación y monitorización de la Comunicación para el Desarrollo y el Cambio Social en España: diseño de indicadores para la medición de su impacto social» (CSO2014-52005-R), cuyo IP es el profesor Víctor M. Marí Sáez, y cuenta, asimismo, con el apoyo del proyecto de I+D «Comunicación especializada y terminografía: usos terminológicos relacionados con los contenidos y perspectivas actuales de la semántica léxica» (FFI2014-54609-P), cuyo IP es el profesor Miguel Casas Gómez. Ambos proyectos están financiados por el Programa Estatal de Fomento de la Investigación Científica y Técnica de Excelencia del Ministerio de Economía y Competitividad de España. La autora pertenece al Grupo de Investigación de Excelencia «Semaínein» (HUM 147) y al Instituto Universitario de Investigación en Lingüística Aplicada de la Universidad de Cádiz (ILA). 
resultados muestran que, aunque son muchos los temas compartidos entre uno y otro corpus, las divergencias son también de gran importancia y es necesario que nos planteemos incluir entre los marcadores temáticos de la disciplina las preocupaciones, valores y principios que se vierten en ambos medios.

Palabras clave: comunicación para el desarrollo y el cambio social, análisis de contenido, análisis de palabras clave, redes sociales, comunicación alternativa, linguiística de corpus.

ABSTRACT: Communication for development and social change (C4D) has experienced in recent years an increase in its importance in the field of communication. However, we lack the indicators that allow us to objectively identify the presence of the C4D in the research on communication in Spain. Faced with this situation, this paper pursues two objectives. On the one hand, it tries to determine the thematic indicators that characterize the discourses on Twitter on CDCS and, on the other hand, it tries to reveal the thematic differences between these discourses and the academic discourse on C4D in Spain. To achieve these objectives, we have made a content analysis based on keywords from a corpus of tweets issued with the hashtag \#comunicambio, and then we compared these results with the keywords of a corpus of scientific articles on C4D published in the main Spanish communication journals. The results show that, although there are many topics shared between the two corpora, the divergences are also of great importance and it is necessary that we consider including among the thematic indicators of the discipline the concerns, values and principles that are poured into both discourses.

Keywords: communication for development and social change, content analysis, keyword analysis, social media, alternative communication, corpus linguistics.

\section{INTRODUCCIÓN}

La importancia estratégica de la comunicación en las organizaciones solidarias ha ido aumentando con el paso de los años. A ello ha contribuido una diversidad de procesos tecnológicos, comunicativos, sociales y políticos, que permiten hoy contemplar el eje comunicativo como una dimensión irrenunciable en la cooperación y en la acción social.

En una primera aproximación se podría decir, siguiendo a Marí y Ceballos (2015: 202), que la comunicación para el desarrollo (Servaes, 2003; Lennie y Tacchi, 2010) y la comunicación para el cambio social (Wilkins, 2000; Gumucio y Tufte, 2006) ofrecen unos marcos teóricos que sirven para identificar y descri- 
bir un campo que tiene que ver con el rol de la comunicación en los esfuerzos estratégicos para superar los problemas colectivos. La comunicación para el desarrollo y el cambio social (CDCS en adelante) es, para estos autores, un campo que se concibe en términos académicos y prácticos. De manera más concreta, Fraser y Restrepo-Estrada (1998: 63) describen el campo como

the use of communication processes, techniques and media to help people toward a full awareness of their situation and their options for change, to resolve conflicts, to work toward consensus, to help people plan actions for change and sustainable development, to help people acquire the knowledge and skills they need to improve their condition and that of society, and to improve the effectiveness of institutions.

El creciente protagonismo de este campo de la comunicación llega acompañado de una amplia diversidad de enfoques teórico-prácticos desde los que se concibe la acción comunicativa. La mayoría de estos enfoques están dominados por un imaginario en el que la comunicación se reduce a la transmisión de información y a la modificación de las conductas. Sin embargo, la CDCS es susceptible de ubicarse en unos imaginarios y marcos teóricos más amplios y abarcadores, que permitan identificar el papel estratégico de la comunicación para la construcción de una ciudadanía solidaria global.

Como señala Marí Sáez (2013: 53), esta rapidez en la expansión de la CDCS en España «contrasta con el olvido y marginación que históricamente ha tenido, hasta convertirse en un fenómeno de moda [...] que se manifiesta en jornadas, publicaciones, proyectos de investigación y grupos en redes sociales que abordan, de un modo u otro, el enfoque de la CDCS». Este fenómeno ha traído consigo que la disciplina, en España, haya llegado a «un punto de inflexión y de crecimiento sin haber consolidado suficientemente el contexto social de la producción científica, el contexto institucional y el contexto epistemológico» (Marí Sáez, 2013: 53) y, por tanto, que este boom pueda ser analizado «más bien, como una implosión del campo; esto es, como una ruptura hacia dentro, debido a la inconsistencia y debilidad con la que fue construido en su fase de institucionalización» (Marí Sáez, 2017: 28).

En este contexto, la investigación que aquí presentamos forma parte de un proyecto interdisciplinar cuyo objetivo es la evaluación del campo de la CDCS en España, a partir del diseño de un dispositivo metodológico que, partiendo de distintos tipos de marcadores, permita mapear la presencia de la CDCS en la investigación sobre comunicación en España, tomando como referencia investigaciones similares impulsadas con éxito en países anglosajones (Lennie y Tacchi, 2013). Consideramos que el hecho de que la expansión de la CDCS no haya estado lo suficientemente cimentada en la producción científica de calidad, en el 
avance institucional ni en el desarrollo epistemológico riguroso hace que sea una necesidad estratégica que este campo sea abordado a través de una investigación rigurosa y seria, que parta de la evaluación de lo realizado y que permita establecer indicadores que traduzcan a un nivel operativo los principios, valores y tradición reflexiva de la CDCS.

Así pues, con este escenario de fondo y teniendo en cuenta la importancia de las redes sociales en la CDCS, el objetivo que nos planteamos en este artículo es contribuir a esta investigación mediante el aporte de una serie de marcadores temáticos que caractericen a la disciplina y que, por tanto, permitan delimitarla y describir cuáles son las ideas principales sobre las que reflexiona. Todo ello es el paso previo para la necesaria evaluación a la que hemos hecho referencia más arriba. En un trabajo anterior (Sánchez-Saus, 2017) analizamos las categorías temáticas predominantes en la reflexión académica sobre CDCS aparecida en las principales revistas de comunicación en España (en aquellos trabajos que Marí y Ceballos (2015) categorizaron como artículos auténticamente sobre CDCS); en este ampliamos el análisis a la que se ha convertido en una de las principales fuentes de textos sobre la materia: las redes sociales, y particularmente en la comunidad digital de debate reunida en torno al hashtag \#comunicambio en la red social Twitter. Esta comunidad nace de la observación sobre cómo dialogan las prácticas comunicativas de las ONG y las ONGD y las reflexiones académicas de profesores universitarios especialistas en el tema. El foro lleva reuniéndose desde 2006 con asiduidad y es especialmente activo en Twitter desde el Congreso Internacional de Comunicación, Sociedad Civil y Cambio Social \#comunicambio, celebrado en la Universitat Jaume I en mayo de $2015 .^{2}$

El resultado de la comparación entre las palabras clave de ambos tipos de textos nos permitirá, por un lado, observar si la inconsistencia científica a la que hemos hecho referencia se traduce en una heterogeneidad temática entre ellos y, por otro, dará pie a reflexionar sobre la necesidad de incluir como indicadores temáticos de la CDCS aquellos que, aunque no estén en la reflexión académica -el medio tradicional, podríamos decir el canónico-, sí que están presentes en textos fundamentales que tratan y discuten sobre la disciplina-las redes sociales, donde se unen las voces de los académicos con la sociedad civil y los activistas.

Para llevar a cabo esta comparación hacemos uso de las herramientas que pone a nuestra disposición la linguiística de corpus (LC). La razón fundamental para emplear esta metodología radica en que la LC nos permite llevar a cabo análisis de contenido de grandes cantidades de texto con rapidez, precisión y consistencia, a un bajo coste, gracias a la automatización de parte del trabajo.

2. El artículo de Erro y Santolino (2015) sobre el desarrollo de este congreso describe los comienzos del foro y la evolución de sus reflexiones. 
La definición de LC es controvertida, ${ }^{3}$ pero por su precisión e interdisciplinariedad, señalamos la que aporta Parodi (2008: 96):

la LC se define, stricto sensu, como una metodología para la investigación de las lenguas y del lenguaje, la cual permite llevar a cabo investigaciones empíricas en contextos auténticos [...]. Desde este enfoque, se estudia información lingüística original y completa, compilada a través de corpus, dado que desde la LC no se apoya la indagación de datos fragmentados, inconexos o de textos incompletos, sino de unidades de sentido y con propósitos comunicativos específicos.

Como señalan Mancera y Pano (2014: 306), los corpus nos «permiten una adecuada representación del discurso en muestras amplias y representativas de textos originales; [...] es posible realizar análisis más amplios y detallados; ofrece mayor fiabilidad en los análisis cuantitativos y cualitativos; los resultados son acumulativos y confrontables con posteriores investigaciones».

De entre las herramientas que pone a nuestra disposición la LC para llevar a cabo análisis de contenido, en esta investigación emplearemos el análisis de palabras clave, ya que, «frente a los análisis de contenido basados en listas de palabras frecuentes, las listas de palabras clave no contienen las más frecuentes, sino aquellas con frecuencias desproporcionadas en relación con la norma. De este modo, los análisis de palabras clave subrayan el cambio o variación y neutralizan las similitudes entre dos corpus» (Duque, 2014: 42) y «permiten el abordaje de corpus extensos sin renunciar a la obtención de características detalladas y relevantes de las representaciones textuales» (Duque, 2014: 69). Con esta comparación, conseguimos que los elementos léxicos más relevantes no se oculten entre las palabras más frecuentes o más repetidas entre los dos textos. Mediante el análisis de palabras clave podremos llevar a cabo un análisis de contenido en el que asumimos que hay una relación entre las repeticiones de una palabra y su relevancia en el discurso y en la construcción de representaciones.

\section{CORPUS Y MÉTODO}

El análisis que presentamos ha sido realizado a partir de dos corpus, ambos compuestos por textos sobre CDCS. El primero de ellos y en el que se centra nuestro análisis es un corpus de 86.394 palabras extraídas de tuits marcados con el hashtag \#comunicambio en la red social Twitter entre los meses de junio de

3. Véase Parodi (2008: esp. 95-99) para un análisis de las distintas visiones que aporta la bibliografía sobre el concepto de lingüística de corpus. 
2015 y febrero de 2017. Hemos evitado los mensajes enviados durante el congreso \#comunicambio de mayo de 2015 para que los contenidos del programa no influyeran en los resultados del análisis. Los tuits marcados con el hashtag \#comunicambio se monitorizaron y extrajeron con la herramienta que proporciona la app Audiense, en combinación con la búsqueda avanzada de Twitter para los tuits anteriores al 1 de enero de 2016. Se han analizado un total de 2.457 tuits y la base de datos cuenta con la participación de 990 cuentas de usuario.

El segundo corpus, que nos servirá de comparación, está formado por los artículos que el análisis bibliométrico llevado a cabo por Marí y Ceballos (2015) determinó como pertenecientes al campo de la CDCS en España en los últimos quince años. Estos son: Tufte (2006), Barranquero (2007 y 2012), Sierra (2000), Sáez Baeza (2009), Ramírez de la Piscina (2010), Marques de Melo (2002), Beltrán (2007), Chaparro (2008 y 2009), Servaes (2012), Fernández Viso (2012), Martínez y Agudiez (2012), Alvarado (2012), Nos, Iranzo y Farné (2012), Herrero y Toledo (2012), Marí (2007), Collado (2008) y Gámez, Fuentes y Nos (2012). Completan esta selección otros tres artículos aparecidos con posterioridad al periodo que analizan Marí y Ceballos (2015) que cumplen con los mismos requisitos bibliométricos: Arroyo (2013), Chaparro (2013) y Travesedo de Castilla (2013). Este segundo corpus está formado por 150.281 palabras.

Para el tratamiento de los corpus hemos empleado el programa AntConc (Anthony, 2017). Con las propias opciones que proporciona el programa, ambos corpus han sido lematizados (uniendo bajo una misma forma todas las variantes morfológicas de una palabra, así como las posibles variantes ortográficas $)^{4}$ y se ha utilizado una stoplist (lista de palabras que se excluyen del análisis) compuesta por palabras gramaticales frecuentes en español (preposiciones, conjunciones, determinantes, verbos auxiliares) y por los términos más usuales en los timelines de Twitter («tweet», «respuesta», «retweet», «seguir», etc.). Asimismo, se han eliminado todos los nombres de usuario, todo ello con el objetivo de que las palabras más frecuentes resultantes del análisis sean palabras de contenido léxico no influidas por la terminología propia de la red social. ${ }^{5}$

Se han empleado fundamentalmente dos tipos de herramientas para el tratamiento de los corpus en AntConc. En primer lugar, hemos extraído las palabras más frecuentes de cada uno de los dos corpus, ordenadas en función del número de veces que aparecen en los textos. En segundo lugar, hemos llevado a cabo

4. Sobre la ortografía en las redes sociales y cómo influye en el tratamiento automático de textos, puede consultarse Mancera y Pano (2014) para el español y Bernicot, Goumi et al. (2014) para el inglés.

5. Pueden consultarse los problemas más comunes vinculados al uso de herramientas informáticas para la automatización de análisis de corpus (lematización, polisemia y homonimia, por ejemplo) en Scott y Tribble (2006: 14 y ss). 
un análisis de palabras clave del corpus extraído de Twitter en comparación con el corpus de textos académicos. Para llevar a cabo esta comparación sin que el resultado se viera influido por las diferencias propias del tipo de discurso (muy formal y académico en un caso, más informal -coloquial incluso- y adaptado a las características propias de Twitter en el otro) la comparación no la hemos realizado con los textos académicos en bruto, sino con la lista de las 1.000 primeras palabras más frecuentes empleadas en los textos académicos sobre CDCs (véase Sánchez-Saus: 2017).

Para calcular el índice de propensión o keyness de cada palabra clave, el programa AntConc lleva incorporada la medida estadística Log Likelihood. ${ }^{6}$ Sus valores de significación son los siguientes:

Tabla 1. Valores de significación de Log Likelihood

\begin{tabular}{|c|}
\hline 95th percentile; $5 \%$ level; $p<0,05 ;$ critical value $=3,84$ \\
\hline 99th percentile; $1 \%$ level; $p<0,01 ;$ critical value $=6,63$ \\
\hline 99.9th percentile; $0,1 \%$ level; $p<0,001 ;$ critical value $=10,83$ \\
\hline 99.99th percentile; $0.01 \%$ level; $p<0,0001 ;$ critical value $=15,13$ \\
\hline
\end{tabular}

En la lista de las primeras 150 palabras clave del corpus de \#comunicambio que aquí analizamos el valor de la keyness es siempre superior a 49, por lo que su significatividad en tanto que palabras clave está lejos de cualquier duda.

Una vez obtenidas las palabras clave, observamos cuáles son las categorías temáticas en torno a las cuales se agrupan, para determinar qué marcadores temáticos caracterizan estos discursos sobre CDCS en Twitter. Secundariamente, comparamos los resultados con las principales categorías temáticas que surgen del corpus académico, para determinar sus convergencias y divergencias.

Ciertas palabas clave solo pueden incluirse en una u otra temática, o en varias, si se da el caso (pues las categorías no son mutuamente excluyentes), si tenemos en cuenta el contexto en el que aparecen. Para ello empleamos la herramienta KWIC (KeyWords in Context) que proporciona igualmente AntConc y que permite observar cómo se han usado en el corpus y rodeadas de qué otras palabras aparecen en su contexto original. Asimismo, el programa nos permite observar la frecuencia de los clusters que nos interesen, esto es, de las combinaciones en las que aparece una palabra determinada. En algunas ocasiones la herramienta

6. Sobre el uso de la medida estadística Log Likelihood para la comparación de corpus, véase Rayson y Garside (2000). 
KWIC nos va a permitir extraer colocación ${ }^{7}$ de las que forma parte la palabra clave, aunque normalmente nos servirá, simplemente, para observar cómo se combinan sintagmáticamente, es decir, a qué palabras aparecen con frecuencia unidas o muy próximas en el corpus.

\section{ANÁLISIS Y RESULTADOS}

Como hemos comentado, para el análisis de las palabras clave vamos a centrarnos en las 150 primeras palabras clave del corpus de \#comunicambio en Twitter y las agruparemos en categorías temáticas.

En cuanto a sus categorías lingüísticas, entre estas palabras clave encontramos sustantivos, verbos y adjetivos, así como nombres propios (topónimos) y los hashtags más relevantes en el corpus analizado.

Tabla 2. Primeras 150 palabras clave del corpus \#comunicambio en Twitter

\begin{tabular}{|r|r|r|r|l|c|c|c|c|l|}
\hline $\mathbf{N}^{\mathbf{}}$ & $\begin{array}{c}\text { Frec. } \\
\text { abs. }\end{array}$ & Frec. rel. & Keyn. & Palabra & $\mathbf{N .}^{\mathbf{0}}$ & $\begin{array}{c}\text { Frec. } \\
\text { abs. }\end{array}$ & Frec. rel. & Keyn. & Palabra \\
\hline $\mathbf{1}$ & 338 & 0,00391 & 888.623 & comunicación & $\mathbf{7 6}$ & 34 & 0,00039 & 89.388 & participar \\
\hline $\mathbf{2}$ & 249 & 0,00288 & 654.637 & mujer & $\mathbf{7 7}$ & 33 & 0,00038 & 86.759 & comenzar \\
\hline $\mathbf{3}$ & 231 & 0,00267 & 607.314 & igualdad & $\mathbf{7 8}$ & 33 & 0,00038 & 86.759 & público \\
\hline $\mathbf{4}$ & 225 & 0,00260 & 591.539 & cambio & $\mathbf{7 9}$ & 33 & 0,00038 & 86.759 & traumático \\
\hline $\mathbf{5}$ & 204 & 0,00236 & 536.329 & social & $\mathbf{8 0}$ & 32 & 0,00037 & 84.130 & humano \\
\hline $\mathbf{6}$ & 195 & 0,00226 & 512.667 & curso & $\mathbf{8 1}$ & 32 & 0,00037 & 84.130 & vivir \\
\hline $\mathbf{7}$ & 143 & 0,00166 & 375.956 & nuevo & $\mathbf{8 2}$ & 31 & 0,00036 & 81.501 & \#altavoz-cambio \\
\hline $\mathbf{8}$ & 136 & 0,00157 & 357.553 & medio & $\mathbf{8 3}$ & 31 & 0,00036 & 81.501 & explicar \\
\hline $\mathbf{9}$ & 134 & 0,00155 & 352.295 & trauma & $\mathbf{8 4}$ & 30 & 0,00035 & 78.872 & compartir \\
\hline $\mathbf{1 0}$ & 118 & 0,00137 & 310.230 & revista & $\mathbf{8 5}$ & 30 & 0,00035 & 78.872 & decir \\
\hline $\mathbf{1 1}$ & 111 & 0,00128 & 291.826 & género & $\mathbf{8 6}$ & 30 & 0,00035 & 78.872 & formación \\
\hline $\mathbf{1 2}$ & 104 & 0,00120 & 273.423 & violencia & $\mathbf{8 7}$ & 30 & 0,00035 & 78.872 & formar \\
\hline $\mathbf{1 3}$ & 97 & 0,00112 & 255.019 & comunicador & $\mathbf{8 8}$ & 30 & 0,00035 & 78.872 & publicar \\
\hline
\end{tabular}

7. Entendemos el término colocación como aquellas «unidades fraseológicas formadas por dos unidades léxicas en relación sintáctica, que no constituyen, por sí mismas, actos de habla ni enunciados; y que, debido a su fijación en la norma, presentan restricciones de combinación establecidas por el uso, generalmente de base semántica» (Corpas Pastor, 1996: 66). 


\begin{tabular}{|c|c|c|c|c|c|c|c|c|c|}
\hline 14 & 92 & 0,00106 & 241.874 & historia & 89 & 29 & 0,00034 & 76.243 & conferencia \\
\hline 15 & 92 & 0,00106 & 241.874 & periodismo & 90 & 29 & 0,00034 & 76.243 & documental \\
\hline 16 & 89 & 0,00103 & 233.987 & narrativo & 91 & 29 & 0,00034 & 76.243 & femenino \\
\hline 17 & 77 & 0,00089 & 202.438 & hablar & 92 & 29 & 0,00034 & 76.243 & gente \\
\hline 18 & 77 & 0,00089 & 202.438 & Madrid & 93 & 29 & 0,00034 & 76.243 & recordar \\
\hline 19 & 77 & 0,00089 & 202.438 & vídeo & 94 & 28 & 0,00032 & 73.614 & acción \\
\hline 20 & 74 & 0,00086 & 194.551 & artículo & 95 & 28 & 0,00032 & 73.614 & discurso \\
\hline 21 & 74 & 0,00086 & 194.551 & blog & 96 & 28 & 0,00032 & 73.614 & marcha \\
\hline 22 & 68 & 0,00079 & 178.776 & paz & 97 & 28 & 0,00032 & 73.614 & silencio \\
\hline 23 & 67 & 0,00078 & 176.147 & \#noesno & 98 & 28 & 0,00032 & 73.614 & trabajo \\
\hline 24 & 66 & 0,00076 & 173.518 & sociedad & 99 & 27 & 0,00031 & 70.985 & abrir \\
\hline 25 & 63 & 0,00073 & 165.631 & prostitución & 100 & 27 & 0,00031 & 70.985 & aula \\
\hline 26 & 62 & 0,00072 & 163.002 & $\begin{array}{l}\text { \#comunica- } \\
\text { igualdad }\end{array}$ & 101 & 27 & 0,00031 & 70.985 & machismo \\
\hline 27 & 62 & 0,00072 & 163.002 & mundo & 102 & 27 & 0,00031 & 70.985 & mirar \\
\hline 28 & 60 & 0,00069 & 157.744 & representar & 103 & 27 & 0,00031 & 70.985 & perder \\
\hline 29 & 59 & 0,00068 & 155.115 & contar & 104 & 27 & 0,00031 & 70.985 & red \\
\hline 30 & 58 & 0,00067 & 152.486 & noticia & 105 & 27 & 0,00031 & 70.985 & taller \\
\hline 31 & 57 & 0,00066 & 149.857 & educación & 106 & 26 & 0,00030 & 68.356 & construir \\
\hline 32 & 57 & 0,00066 & 149.857 & seminario & 107 & 26 & 0,00030 & 68.356 & cooperación \\
\hline 33 & 57 & 0,00066 & 149.857 & $\begin{array}{l}\text { \#somos- } \\
\text { iguales }\end{array}$ & 108 & 26 & 0,00030 & 68.356 & ejemplo \\
\hline 34 & 56 & 0,00065 & 147.228 & interesante & 109 & 26 & 0,00030 & 68.356 & planeta \\
\hline 35 & 56 & 0,00065 & 147.228 & periodista & 110 & 26 & 0,00030 & 68.356 & representación \\
\hline 36 & 54 & 0,00063 & 141.969 & \#comigualdad & 111 & 25 & 0,00029 & 65.727 & buscar \\
\hline 37 & 54 & 0,00063 & 141.969 & cultura & 112 & 25 & 0,00029 & 65.727 & festival \\
\hline 38 & 53 & 0,00061 & 139.340 & ONG & 113 & 25 & 0,00029 & 65.727 & partir \\
\hline 39 & 51 & 0,00059 & 134.082 & proyecto & 114 & 25 & 0,00029 & 65.727 & trabajar \\
\hline 40 & 50 & 0,00058 & 131.453 & campaña & 115 & 25 & 0,00029 & 65.727 & solidaridad \\
\hline 41 & 50 & 0,00058 & 131.453 & premio & 116 & 24 & 0,00028 & 63.098 & activismo \\
\hline 42 & 49 & 0,00057 & 128.824 & agencia & 117 & 24 & 0,00028 & 63.098 & agenda \\
\hline 43 & 48 & 0,00056 & 126.195 & comunicar & 118 & 24 & 0,00028 & 63.098 & charlar \\
\hline 44 & 47 & 0,00054 & 123.566 & altavoz & 119 & 24 & 0,00028 & 63.098 & escuchar \\
\hline
\end{tabular}




\begin{tabular}{|c|c|c|c|c|c|c|c|c|c|}
\hline 45 & 47 & 0,00054 & 123.566 & futuro & 120 & 24 & 0,00028 & 63.098 & guerra \\
\hline 46 & 46 & 0,00053 & 120.937 & crear & 121 & 24 & 0,00028 & 63.098 & intención \\
\hline 47 & 46 & 0,00053 & 120.937 & debatir & 122 & 24 & 0,00028 & 63.098 & libro \\
\hline 48 & 46 & 0,00053 & 120.937 & desarrollo & 123 & 24 & 0,00028 & 63.098 & memoria \\
\hline 49 & 46 & 0,00053 & 120.937 & África & 124 & 24 & 0,00028 & 63.098 & necesitar \\
\hline 50 & 45 & 0,00052 & 118.308 & cine & 125 & 24 & 0,00028 & 63.098 & presentar \\
\hline 51 & 45 & 0,00052 & 118.308 & hombre & 126 & 24 & 0,00028 & 63.098 & reflexionar \\
\hline 52 & 44 & 0,00051 & 115.679 & bueno & 127 & 23 & 0,00027 & 60.468 & aprender \\
\hline 53 & 44 & 0,00051 & 115.679 & concienciar & 128 & 23 & 0,00027 & 60.468 & citar \\
\hline 54 & 44 & 0,00051 & 115.679 & persona & 129 & 23 & 0,00027 & 60.468 & congreso \\
\hline 55 & 44 & 0,00051 & 115.679 & político & 130 & 23 & 0,00027 & 60.468 & conocer \\
\hline 56 & 43 & 0,00050 & 113.050 & cambiar & 131 & 23 & 0,00027 & 60.468 & creer \\
\hline 57 & 43 & 0,00050 & 113.050 & enfoque & 132 & 23 & 0,00027 & 60.468 & encuentro \\
\hline 58 & 43 & 0,00050 & 113.050 & hambre & 133 & 23 & 0,00027 & 60.468 & paso \\
\hline 59 & 43 & 0,00050 & 113.050 & internacional & 134 & 23 & 0,00027 & 60.468 & saber \\
\hline 60 & 41 & 0,00047 & 107.792 & machista & 135 & 22 & 0,00025 & 57.839 & Euskadi \\
\hline 61 & 40 & 0,00046 & 105.163 & empezar & 136 & 22 & 0,00025 & 57.839 & realidad \\
\hline 62 & 40 & 0,00046 & 105.163 & feminista & 137 & 22 & 0,00025 & 57.839 & tratamiento \\
\hline 63 & 40 & 0,00046 & 105.163 & vida & 138 & 21 & 0,00024 & 55.210 & espacio \\
\hline 64 & 39 & 0,00045 & 102.533 & imagen & 139 & 21 & 0,00024 & 55.210 & Facebook \\
\hline 65 & 38 & 0,00044 & 99.904 & derecho & 140 & 21 & 0,00024 & 55.210 & programa \\
\hline 66 & 38 & 0,00044 & 99.904 & feminismo & 141 & 21 & 0,00024 & 55.210 & unir \\
\hline 67 & 38 & 0,00044 & 99.904 & información & 142 & 21 & 0,00024 & 55.210 & violación \\
\hline 68 & 38 & 0,00044 & 99.904 & luchar & 143 & 20 & 0,00023 & 52.581 & desigualdad \\
\hline 69 & 36 & 0,00042 & 94.646 & España & 144 & 20 & 0,00023 & 52.581 & estereotipo \\
\hline 70 & 36 & 0,00042 & 94.646 & majo & 145 & 20 & 0,00023 & 52.581 & herramienta \\
\hline 71 & 36 & 0,00042 & 94.646 & refugiado & 146 & 20 & 0,00023 & 52.581 & voz \\
\hline 72 & 36 & 0,00042 & 94.646 & víctima & 147 & 19 & 0,00022 & 49.952 & actualidad \\
\hline 73 & 35 & 0,00041 & 92.017 & romper & 148 & 19 & 0,00022 & 49.952 & arma \\
\hline 74 & 34 & 0,00039 & 89.388 & experiencia & 149 & 19 & 0,00022 & 49.952 & ayuda \\
\hline 75 & 34 & 0,00039 & 89.388 & jornada & 150 & 19 & 0,00022 & 49.952 & ciudadano \\
\hline
\end{tabular}


Las categorías temáticas en torno a las que se agrupan las palabras clave de \#comunicambio son varias:

1. Palabras que forman el propio término «comunicación para el desarrollo y el cambio social»: «comunicación», «cambio», «social», «desarrollo». Las tres primeras se encuentran entre las cinco primeras palabras clave del corpus; «desarrollo», en cambio, no aparece hasta el cuadragésimo octavo, con una frecuencia cinco veces menor que «social».

2. Cuestiones de género e igualdad: «mujer», «igualdad», «género», «trauma», «violencia», «prostitución», «machista», «feminista», «feminismo», «traumático», «femenino», «violación», así como los hashtags \#noesno, \#comunicaigualdad, \#somosiguales y \#comigualdad. El término «igualdad» aparece dieciséis veces en el cluster «igualdad de género» (que conforma una colocación en español) y tres en «igualdad entre hombres y mujeres»y, en general, cuando se usa sin complementos hace referencia a este sentido de igualdad. En el caso de «trauma», la incluimos en este tema después de haber empleado la herramienta KWIC y calculado la frecuencia de los clusters en los que aparece, que nos permiten observar que ha aparecido diecisiete veces seguido directamente de «femenino» y en doce ocasiones el término «violación» aparece en el mismo tuit. Con «traumático» hemos seguido el mismo proceso: en ocho ocasiones está combinado con «cultura» y en seis, con «memoria», siempre referido a la violencia contra la mujer en la sociedad y sus consecuencias. Igualmente, incluimos «violencia» dentro del tema de la igualdad de género porque en sesenta y dos casos se combina en el discurso con «contra la mujer» o «de género» (en ambos casos se trata de una colocación).

3 Comunicación en general: «hablar», «comunicar», «debatir», «explicar», «discurso», «decir», «charlar», «escuchar».

4. Contexto académico y universitario: «curso», «revista», «artículo», «seminario», «jornada», «publicar», «enfoque», «conferencia», «aula», «presentar», «congreso», «taller», «encuentro». El uso de Twitter está muy vinculado a la descripción del contexto directo en el que se encuentra el emisor. La comunidad formada en torno a \#comunicambio se reúne con frecuencia en conferencias, cursos, seminarios y congresos, por lo que era de esperar la preponderancia de esta categoría.

5. Narración: «historia», «narrativo», «contar», «experiencia». ${ }^{8}$

8. Resultan muy interesantes, en relación con la importancia de la narración en la comunicación para el cambio social y, particularmente, de la narración transmedia, las reflexiones que desarrollan García López y Simancas González (2016). 
6. Medios de comunicación: «medio», «comunicador», «periodismo», «artículo», «noticia», «agencia», «periodista», «información», «publicar», «actualidad». Particularmente, con los medios digitales y las redes sociales están relacionados «blog» $\mathrm{y}$ «Facebook». Los términos «VOz» $\mathrm{y}$ «altavoz» son importantes también en esta categoría, pues suelen aparecer relacionados con el papel de los medios para «dar voz» a los que no la tienen o «ser altavoz» de estos.

7. El cambio y lo nuevo: «nuevo», «cambio», «cambiar», «crear», «futuro», «romper». En esta última palabra, el análisis de clusters revela que en catorce casos aparece en combinación con «el silencio»; por ello lo incluimos en la categoría «cambio». La palabra «futuro» suele ir asociada a la «transformación», igualmente, en usos como «vosotros sois el futuro» $\mathrm{O}$ «construir un futuro mejor».

8. Sociedad y ciudadanía: «sociedad», «mundo», «cultura», «hombre», «persona», «público», «humano», «red», «ciudadano».

9. Movimientos sociales y participación: «ONG», «proyecto», «concienciar», «derecho», «acción», «activismo», «participar», «solidaridad», «cooperación», «refugiado», «marcha», «ayuda».

10. Educación: «educación», «formar», «formación», «aprender».

11. África: surge como una categoría en sí misma, transversal a muchos temas, como revela el que en el contexto se vincule a «representación», «imagen», «imaginario», «narrativa», «discursos», «paz», «desarrollo», «música» o «tradición». La connotación suele ser positiva y relacionada también con el cambio, en el sentido de que deben romperse los estereotipos («hambre», «guerra») y se debe comunicar una imagen distinta en los medios.

12. Palabras relacionadas con la lucha: «luchar», «arma», «guerra». Al contrario de lo que ocurría con el término «violencia», asociado, como hemos visto, a la violencia machista, el concepto «luchar» se emplea en el corpus de manera claramente positiva: se lucha «contra la desigualdad», «por la igualdad» «por el cambio», «por la mujer», «por los derechos», «contra la violencia». Ocurre algo parecido con «arma», que se emplea también con un sentido figurado: «arma de transformación», «arma para el cambio», «arma de desinformación». Asimismo, «arma» aparece en cuatro ocasiones en combinación con «guerra» para hablar del hambre y las violaciones: «hambre como arma de guerra», «violación como arma de guerra».

Comparemos ahora estas categorías y su relevancia con las que resultan del análisis llevado a cabo sobre el corpus de artículos académicos sobre CDCs (Sánchez-Saus: 2017). 
Tabla 3. Primeras 150 palabras clave del corpus académico sobre CDCS

\begin{tabular}{|c|c|c|c|c|c|c|c|c|c|}
\hline N. ${ }^{o}$ & $\begin{array}{c}\text { Frec. } \\
\text { abs. }\end{array}$ & Frec. rel. & Keyness & Palabra & N. ${ }^{\circ}$ & $\begin{array}{c}\text { Frec. } \\
\text { abs. }\end{array}$ & Frec. rel. & Keyness & Palabra \\
\hline 1 & 873 & 0,00581 & 2498121 & desarrollo & 76 & 103 & 0,00069 & 250335 & década \\
\hline 2 & 1460 & 0,00972 & 2249971 & comunicación & 77 & 95 & 0,00063 & 250152 & colectivo \\
\hline 3 & 941 & 0,00626 & 2043377 & social & 78 & 90 & 0,00060 & 247169 & recurso \\
\hline 4 & 682 & 0,00454 & 1971276 & medio & 79 & 48 & 0,00032 & 246194 & necesitar \\
\hline 5 & 484 & 0,00322 & 1426110 & cambio & 80 & 109 & 0,00073 & 245373 & mundial \\
\hline 6 & 492 & 0,00327 & 1348272 & nuevo & 81 & 127 & 0,00085 & 242945 & necesario \\
\hline 7 & 410 & 0,00273 & 1301699 & político & 82 & 167 & 0,00111 & 240825 & tecnología \\
\hline 8 & 252 & 0,00168 & 1057129 & formar & 83 & 83 & 0,00055 & 240131 & definir \\
\hline 9 & 199 & 0,00132 & 1004164 & deber & 84 & 80 & 0,00053 & 234472 & vasco \\
\hline 10 & 161 & 0,00107 & 808628 & alternativo & 85 & 81 & 0,00054 & 234041 & llevar \\
\hline 11 & 153 & 0,00102 & 716931 & comunitario & 86 & 90 & 0,00060 & 230442 & encontrar \\
\hline 12 & 186 & 0,00124 & 709119 & comunicativo & 87 & 104 & 0,00069 & 228830 & persona \\
\hline 13 & 140 & 0,00093 & 702162 & sentir & 88 & 118 & 0,00079 & 223379 & perspectiva \\
\hline 14 & 289 & 0,00192 & 632566 & cultural & 89 & 39 & 0,00026 & 222562 & falta \\
\hline 15 & 114 & 0,00076 & 623019 & unir & 90 & 97 & 0,00065 & 221482 & enfoque \\
\hline 16 & 111 & 0,00074 & 606057 & interesar & 91 & 99 & 0,00066 & 221168 & desarrollar \\
\hline 17 & 183 & 0,00122 & 597936 & ciudadano & 92 & 74 & 0,00049 & 218543 & ayudar \\
\hline 18 & 265 & 0,00176 & 575832 & partir & 93 & 130 & 0,00087 & 218162 & acción \\
\hline 19 & 126 & 0,00084 & 563617 & práctico & 94 & 46 & 0,00031 & 218155 & venir \\
\hline 20 & 153 & 0,00102 & 536917 & humano & 95 & 80 & 0,00053 & 216950 & poner \\
\hline 21 & 94 & 0,00063 & 536432 & libertad & 96 & 131 & 0,00087 & 215289 & necesidad \\
\hline 22 & 298 & 0,00198 & 528541 & público & 97 & 43 & 0,00029 & 213905 & demandar \\
\hline 23 & 114 & 0,00076 & 519269 & participativo & 98 & 145 & 0,00096 & 212357 & espacio \\
\hline 24 & 113 & 0,00075 & 513974 & relacionar & 99 & 113 & 0,00075 & 209962 & teoría \\
\hline 25 & 93 & 0,00062 & 504382 & base & 100 & 179 & 0,00119 & 209508 & publicidad \\
\hline 26 & 209 & 0,00139 & 480123 & radio & 101 & 107 & 0,00071 & 207928 & idea \\
\hline 27 & 236 & 0,00157 & 479435 & modelo & 102 & 61 & 0,00041 & 207349 & sostenible \\
\hline 28 & 83 & 0,00055 & 473658 & mercado & 103 & 86 & 0,00057 & 205463 & considerar \\
\hline 29 & 170 & 0,00113 & 464103 & movimiento & 104 & 55 & 0,00037 & 205117 & distinto \\
\hline
\end{tabular}




\begin{tabular}{|c|c|c|c|c|c|c|c|c|c|}
\hline 30 & 118 & 0,00079 & 463574 & emisora & 105 & 69 & 0,00046 & 204487 & paradigma \\
\hline 31 & 181 & 0,00120 & 453836 & económico & 106 & 82 & 0,00055 & 203944 & teórico \\
\hline 32 & 97 & 0,00065 & 439012 & debate & 107 & 40 & 0,00027 & 201936 & indígena \\
\hline 33 & 104 & 0,00069 & 438161 & resultar & 108 & 70 & 0,00047 & 201694 & tomar \\
\hline 34 & 271 & 0,00180 & 428526 & proceso & 109 & 65 & 0,00043 & 199713 & nación \\
\hline 35 & 157 & 0,00104 & 413891 & derecho & 110 & 62 & 0,00041 & 198571 & grande \\
\hline 36 & 173 & 0,00115 & 413026 & propio & 111 & 123 & 0,00082 & 198463 & concepto \\
\hline 37 & 246 & 0,00164 & 388496 & país & 112 & 56 & 0,00037 & 197246 & beneficio \\
\hline 38 & 180 & 0,00120 & 383221 & proyecto & 113 & 108 & 0,00072 & 197211 & ciudadanía \\
\hline 39 & 72 & 0,00048 & 380018 & condicionar & 114 & 39 & 0,00026 & 196422 & comprometido \\
\hline 40 & 83 & 0,00055 & 377836 & existir & 115 & 55 & 0,00037 & 195989 & bueno \\
\hline 41 & 148 & 0,00098 & 374744 & crítico & 116 & 57 & 0,00038 & 195309 & parecer \\
\hline 42 & 173 & 0,00115 & 365584 & comunidad & 117 & 63 & 0,00042 & 194900 & valorar \\
\hline 43 & 85 & 0,00057 & 354078 & convertir & 118 & 54 & 0,00036 & 194792 & masivo \\
\hline 44 & 162 & 0,00108 & 347762 & local & 119 & 66 & 0,00044 & 194147 & reconocer \\
\hline 45 & 186 & 0,00124 & 347328 & año & 120 & 42 & 0,00028 & 193219 & latino \\
\hline 46 & 66 & 0,00044 & 346454 & causa & 121 & 75 & 0,00050 & 193122 & construir \\
\hline 47 & 151 & 0,00100 & 338926 & problema & 122 & 61 & 0,00041 & 192778 & compartir \\
\hline 48 & 146 & 0,00097 & 328326 & dar & 123 & 127 & 0,00085 & 192401 & objetivo \\
\hline 49 & 235 & 0,00156 & 327205 & cultura & 124 & 77 & 0,00051 & 190030 & mejorar \\
\hline 50 & 61 & 0,00041 & 324248 & temer & 125 & 66 & 0,00044 & 189520 & contribuir \\
\hline 51 & 67 & 0,00045 & 316469 & estructurar & 126 & 43 & 0,00029 & 189109 & querer \\
\hline 52 & 98 & 0,00065 & 308315 & producir & 127 & 87 & 0,00058 & 187688 & mediático \\
\hline 53 & 54 & 0,00036 & 308163 & prensa & 128 & 134 & 0,00089 & 187439 & global \\
\hline 54 & 63 & 0,00042 & 306337 & disciplina & 129 & 45 & 0,00030 & 185986 & centrar \\
\hline 55 & 93 & 0,00062 & 301375 & tratar & 130 & 44 & 0,00029 & 185978 & diverso \\
\hline 56 & 66 & 0,00044 & 297599 & surgir & 131 & 82 & 0,00055 & 185888 & comercial \\
\hline 57 & 52 & 0,00035 & 296750 & privar & 132 & 92 & 0,00061 & 184245 & democracia \\
\hline 58 & 78 & 0,00052 & 296722 & permitir & 133 & 59 & 0,00039 & 184001 & justicia \\
\hline 59 & 65 & 0,00043 & 295506 & implicar & 134 & 82 & 0,00055 & 183620 & globalización \\
\hline 60 & 142 & 0,00094 & 294965 & programa & 135 & 62 & 0,00041 & 181951 & pasar \\
\hline
\end{tabular}




\begin{tabular}{|r|r|r|r|l|l|l|l|l|l|}
\hline $\mathbf{6 1}$ & 154 & 0,00102 & 293959 & participación & $\mathbf{1 3 6}$ & 54 & 0,00036 & 181571 & actor \\
\hline $\mathbf{6 2}$ & 295 & 0,00196 & 292690 & sociedad & $\mathbf{1 3 7}$ & 82 & 0,00055 & 180662 & utilizar \\
\hline $\mathbf{6 3}$ & 91 & 0,00061 & 285623 & sida & $\mathbf{1 3 8}$ & 33 & 0,00022 & 179418 & referir \\
\hline $\mathbf{6 4}$ & 84 & 0,00056 & 282956 & vivir & $\mathbf{1 3 9}$ & 59 & 0,00039 & 178861 & plantear \\
\hline $\mathbf{6 5}$ & 89 & 0,00059 & 282224 & señalar & $\mathbf{1 4 0}$ & 83 & 0,00055 & 177780 & popular \\
\hline $\mathbf{6 6}$ & 105 & 0,00070 & 281207 & diferente & $\mathbf{1 4 1}$ & 73 & 0,00049 & 177372 & generar \\
\hline $\mathbf{6 7}$ & 132 & 0,00088 & 277772 & organización & $\mathbf{1 4 2}$ & 48 & 0,00032 & 177215 & constituir \\
\hline $\mathbf{6 8}$ & 81 & 0,00054 & 273899 & democrático & $\mathbf{1 4 3}$ & 31 & 0,00021 & 176908 & agencia \\
\hline $\mathbf{6 9}$ & 55 & 0,00037 & 266863 & significar & $\mathbf{1 4 4}$ & 31 & 0,00021 & 176908 & conferencia \\
\hline $\mathbf{7 0}$ & 75 & 0,00050 & 265689 & ONG & $\mathbf{1 4 5}$ & 77 & 0,00051 & 176846 & transformación \\
\hline $\mathbf{7 1}$ & 142 & 0,00094 & 261188 & internacional & $\mathbf{1 4 6}$ & 65 & 0,00043 & 176784 & alcanzar \\
\hline $\mathbf{7 2}$ & 118 & 0,00079 & 259283 & estrategia & $\mathbf{1 4 7}$ & 35 & 0,00023 & 174417 & solidario \\
\hline $\mathbf{7 3}$ & 72 & 0,00048 & 258540 & favorecer & $\mathbf{1 4 8}$ & 32 & 0,00021 & 173771 & nivel \\
\hline $\mathbf{7 4}$ & 106 & 0,00071 & 256613 & tecnológico & $\mathbf{1 4 9}$ & 87 & 0,00058 & 173016 & posibilidad \\
\hline $\mathbf{7 5}$ & 88 & 0,00059 & 251941 & masa & $\mathbf{1 5 0}$ & 47 & 0,00031 & 172435 & comenzar \\
\hline
\end{tabular}

Lo primero que llama la atención entre estas primeras 150 palabras clave del corpus académico sobre CDCS es la total ausencia de términos que hagan referencia a cuestiones de género, frente a la preponderancia de esta categoría en el corpus de \#comunicambio. En este segundo corpus la palabra «género» aparece en la posición 7.508 de la lista de palabras clave, con frecuencia 35 y keyness 0,555 (muy por debajo de los niveles de significación), y en la posición 430 en la lista de palabras frecuentes; la palabra «mujer» ni siquiera aparece entre las palabras clave y en las listas de frecuencia está en la posición 546, con frecuencia 28. Por su parte, «igualdad» está en la posición 176 entre las palabras clave, con frecuencia 66 y keyness 156.837 , pero si observamos el contexto en el que aparece nos damos cuenta de que se emplea en un sentido mucho menos restringido que en el corpus de \#comunicambio: se hace referencia a la igualdad «de oportunidades», «de derechos», «de acceso»y, también, «de género» (aunque este cluster aparece solo en dos ocasiones).

Tampoco tiene presencia la categoría relacionada con la narración: «historia» (puesto 1.291, frecuencia 68, keyness 21.433), «contar» (puesto 643, frecuencia 34 , keyness 46.456) y «narrativo» (puesto 1.411, frecuencia 11, keyness 18.649) distan mucho de tener una relevancia semejante a la que tienen para \#comunicambio. 
Ocurre lo mismo con la categoría vinculada a África. Este topónimo no aparece en la lista de palabras clave en textos académicos hasta el puesto 121 , con frecuencia 18 y keyness 24.390 .

Igualmente, palabras con tanta fuerza como las relacionadas con la lucha están ausentes en la lista que presentamos del segundo corpus, aunque en este caso la diferencia entre uno y otro es menor: «luchar» aparece en el corpus académico en la posición 187, con frecuencia 39 y keyness 149.375. «Arma» y «guerra» sí que quedan fuera de las primeras 1.000 palabras clave en este segundo.

No sorprende que la categoría relacionada con el mundo académico y la universidad apenas tenga representación en esta lista (solo aparece «conferencia»), dado que en estos textos no existe esa relación con la descripción directa de los momentos en los que la comunidad se reúne que sí se da en el uso del hashtag \#comunicambio en Twitter.

Otras categorías son comunes a los dos corpus, como las relacionadas con la sociedad y la ciudadanía, los movimientos sociales y la participación, el cambio y los medios de comunicación, aunque, en general, estas cuentan aquí con un número mayor de términos y están posicionados más arriba que en el primer corpus.

Una categoría presente aquí que no estaba en el primer corpus es la vinculada a la economía: «mercado», «económico», «recurso», «comercial».

Por otro lado, es considerable el número de verbos en este segundo corpus (53, frente a los 37 del corpus de \#comunicambio), sobre todo de verbos causati$\operatorname{vos}$ («formar», «convertir», «producir», «favorecer», «desarrollar», «construir»), de proceso mental («interesar», «considerar», «valorar», «reconocer») y de proceso relacional («unir», «relacionar», «resultar», «condicionar», «implicar», «significar», «definir»). Sin duda, esto se debe al tipo de texto analizado: artículos formulados en un estilo formal, con largos párrafos en los que se desarrollan conceptos y se ponen en relación, frente al estilo más informal y directo de la comunicación en redes sociales, y particularmente en Twitter, donde es mucho más frecuente el empleo de sustantivos, de verbos de proceso verbal («decir», «hablar», «charlar», «debatir», «contar»...) y de verbos de acción («empezar», «comenzar», «luchar», «romper», «mirar»). Podríamos determinar, por tanto, en el corpus académico, una última categoría vinculada a los verbos empleados para la reflexión académica. 


\section{CONCLUSIONES}

La situación de la CDCS en España ha sido descrita como en fase de implosión, causada por la inconsistencia de la reflexión científica sobre ella desde que surgió como disciplina en nuestro país. En este artículo hemos querido contribuir a la evaluación de lo realizado dentro de esta disciplina analizando los marcadores temáticos que caracterizan a una de las principales fuentes de reflexión sobre la materia: las redes sociales, y compararlos con aquellos que surgen en la producción académica sobre el tema, poniendo de relieve las divergencias que existen y, al mismo tiempo, la necesidad de que desde la CDCS se preste atención también a lo que se discute fuera de los artículos académicos.

Concretamente, aquí nos hemos centrado en los marcadores temáticos a partir de las categorías que reúnen a las principales palabras clave de los tuits recogidos bajo el hashtag \#comunicambio en Twitter. Es muy significativa en este corpus la relevancia de la categoría relacionada con la igualdad de género y la violencia machista. Los términos «mujer»e «igualdad» son las dos principales palabras clave del corpus, solo precedidas por «comunicación»; asimismo, los cuatro únicos hashtags que se encuentran entre las primeras 150 palabras clave se refieren a esta temática. Es por ello sorprendente que en la comparación que hemos llevado a cabo con el corpus de textos académicos sobre CDCS este tema esté casi por completo ausente.

Otras categorías con menor importancia que las cuestiones de género en \#comunicambio, aunque con una presencia significativa, son las referidas a los medios de comunicación, la necesidad del cambio y la transformación sociales, la sociedad y la ciudadanía, la participación y la movilización ciudadanas. Todas ellas aparecen igualmente en el corpus académico que hemos empleado de comparación. No están presentes en este, en cambio, la educación y la realidad social en África. Además, es interesante el uso figurado y connotativamente positivo que se hace en el corpus de \#comunicambio de ciertos términos relacionados con la violencia («luchar»y «arma»). Asimismo, la relevancia de los términos relacionados con la narración revela el modo en que los discursos son transmitidos: a través de la narración de experiencias y, no tanto, como se observa en el corpus académico, a partir de la reflexión teórica.

Nos encontramos, por tanto, con una serie de marcadores que son comunes a los dos tipos de discurso sobre CDCS, pero también con significativas divergencias, sin duda causadas por la inconsistencia de la disciplina en fases anteriores. Aunque es evidente que las redes sociales tienen una consideración inferior que las revistas académicas, en cuanto medio para la reflexión científica, no creemos que tenga sentido dejar de lado las preocupaciones, valores y principios que en ellas se revelan. 


\section{REFERENCIAS BIBLIOGRÁFICAS}

Alvarado López, M. C. (2012): «La publicidad en el marco de la comunicación para el desarrollo: hacia un nuevo modelo de publicidad para el cambio social», CIC. Cuadernos de Información y Comunicación, 17: 191-207. http://dx.doi.org/10.5209/rev_CIYC.2012.v17.39264.

Anthony, L. (2017): AntConc (versión 3.4.1) [software informático]. Tokio: Waseda University. Recuperado de: https://goo.gl/T1DD7X.

Arroyo Almaraz, I. (2013): «El valor de las ideas para el cambio social: Publicidad social, biopolítica y neuroética», CIC. Cuadernos de Información y Comunicación, 18: 223-241. http://dx.doi.org/10.5209/rev_CIYC.2013. V18.41819.

Barranquero, A. (2007): «Concepto, instrumentos y desafíos de la educomunicación para el cambio social», Comunicar, 29(XV): 115-120. Recuperado de: https://goo.gl/ALC164.

- (2012): «Comunicación, cambio social y ONG en España. Pistas para profundizar en la cultura de la cooperación desde los nuevos movimientos comunicacionales. El caso del 15M», Commons. Revista de Comunicación y Ciudadanía Digital, 3(1): 5-33. Recuperado de: https://goo.gl/Z0XAr0.

Beltrán Salmón, L. R. (2007): «Comunicación para la democracia e Iberoamérica. Memoria y retos del futuro», Revista TELOS, 72. Recuperado de: https://goo.gl/xJ4krX.

Bernicot, J., Goumi, A., Bert-Erboul, A. y Volckaert-Legrier, O. (2014): «How do skilled and less-skilled spellers write text messages? A longitudinal study», Journal of Computer Assisted Learning, 30: 559-576.

Chaparro, M. (2008): «Los escenarios europeo y latinoamericano. Medios comunitarios y ciudadanos», Revista TELOS, 74. Recuperado de: https://goo. $\mathrm{gl} / \mathrm{NclQQR}$.

- (2009): «Comunicación y desarrollo. Retos para un nuevo periodismo», Revista TELOS, 81. Recuperado de: https://goo.gl/K0Chm9.

- (2013): «La comunicación del desarrollo. Construcción de un imaginario perverso», Revista TELOS, 94: 31-42. Recuperado de: https://goo.gl/nyUv1t.

Collado Campaña, F. (2008): «La influencia de las radios y las televisiones comunitarias en la construcción de la ciudadanía», Ámbitos. Revista Andaluza de Comunicación, 17: 209-224. Recuperado de: https://goo.gl/ByUNFN.

Corpas Pastor, G. (1996): Manual de fraseología española. Madrid: Gredos.

DuQUE, E. (2014): «Análisis de contenido mediante análisis de palabras clave: La representación de los participantes en los discursos de Esperanza Aguirre», Mediaciones Sociales. Revista de Ciencias Sociales y de la Comunicación, 13: 39-73. http://dx.doi.org/10.5209/rev_MESO.2014.n13.49432. 
Erro, J. y Santolino, M. (2015, 30 de julio): «Congreso \#comunicambio: Los debates en torno a la comunicación y la solidaridad», Pueblos. Revista de Información y Debate. Recuperado de: https://goo.gl/oVkVMR.

Fernández Viso, A. (2012): «Historia de una travesía inconclusa: la comunicación para el desarrollo y el cambio social en la investigación y la docencia universitarias en España», CIC. Cuadernos de Información y Comunicación, 17: 41-62. Recuperado de: https://goo.gl/EPXO4g.

Fraser, C. y Restrepo-Estrada, S. (1998): Communicating for Development: Human Change for Survival, Londres: I. B. Tauris Publishers.

Gámez Fuentes, M. J. y Nos, E. (2012): «Comunicación para la igualdad en el nuevo EEES: fundamentación crítica para el cambio social», Estudios sobre el Mensaje Periodístico, 18, núm. especial octubre: 325-335. http://dx.doi. org/10.5209/rev_ESMP.2012.v18.40986.

García López, M. y Simancas González, E. (2016): «La lucha está en el relato. Movimientos sociales, narrativas transmedia y cambio social», Cultura, Lenguaje y Representación / Culture, language and representation, XV: 139-151. http://dx.doi.org/10.6035/clr.2016.15.9.

Gumucio, A. y TufTe, T. (2006) (eds.): Communication for Social Change Anthology: Historical and Contemporary readings, Nueva Jersey: CFSC Consortiu.

Herrero, J. C. y Toledo Chávarri, A. (2012): «La profesionalización de la comunicación para el desarrollo: relaciones entre la teoría y la práctica», CIC. Cuadernos de Información y Comunicación, 17: 255-266. http://dx.doi. org/10.5209/rev_CIYC.2012.v17.39267.

LENNIE, J. У TACCHI, J. (2010): Evaluating communication for development: Trends, challenges and approaches. Draft report on a literature review and consultations conduced for the project: UN Inter-agency Research, Monitoring and Evaluation Resource Pack for Communication for Development Programs. Nueva York: UNICEF.

- (2013): Evaluating Communication for Development. A framework for social change. Londres: Routledge.

Mancera Rueda A. y Pano Alamán, A. (2014): «Las redes sociales como corpus de estudio para el análisis del discurso mediado por ordenador», Humanidades Digitales: desafíos, logros y perspectivas de futuro. Janus, Anexo 1: 305-315. Recuperado de: https://goo.gl/zUeP2a.

Marí SÁEz, V. (2007): «Contra la evaporación de la dimensión política de la comunicación. Movimientos sociales, ONG y usos de Internet», Zer, Revista de Estudios de Comunicación, 22: 453-471. Recuperado de: https://goo.gl/ GeUL1U. 
- (2013): «Comunicación, desarrollo y cambio social en España: entre la institucionalización y la implosión del campo», Commons, Revista de Comunicación y Ciudadanía Digital, 2(2): 39-64. Recuperado de: https:// goo.gl/hHws66.

- (2017): «Historia de la comunicación para el desarrollo y el cambio social en España. Marginalidad, institucionalización e implosión de un campo en crecimiento», Telos, 106, 27-38.

Marí Sáez, V. y Ceballos Castro, G. (2015): «Análisis bibliométrico sobre "Comunicación, Desarrollo y Cambio Social" en las diez primeras revistas de comunicación de España», Cuadernos.info, 37: 201-212. http://dx.doi. org/10.7764/cdi.37.828

Marques De Melo, J. (2002): «Exclusión comunicacional y democracia mediática: dilema brasileño en el umbral de la Sociedad de la Información». Revista TELOS, 51.

Martínez Gómez, R. y Agudiez, P. (2012): «Comunicación para el desarrollo humano: buscando la transformación social». CIC. Cuadernos de Información y Comunicación, 17: 79-106. http://dx.doi.org/10.5209/rev_CIYC.2012. v17.39259.

Nos, E., Iranzo, A. y FARné, A. (2012): «La eficacia cultural de la comunicación de las ONGD: los discursos de los movimientos sociales actuales como revisión». CIC. Cuadernos de Información y Comunicación, 17: 209-237. http://dx.doi.org/10.5209/rev_CIYC.2012.v17.39265.

PAROdi, G. (2008): «Lingüística de corpus: una introducción al ámbito», RLA. Revista de Lingüística Teórica y Aplicada, 46(1): 93-119. https://dx.doi. org/10.4067/S0718-48832008000100006

Ramírez de la Piscina Martínez, T. (2010): «País Vasco, laboratorio de comunicación alternativa. Compilación de las experiencias más interesantes», Revista Latina de Comunicación Social, 65: 310-324. http:// dx.doi.org/10.4185/RLCS-65-2010-902-310-324.

Rayson, P. y GaRside, R. (2000): «Comparing corpora using frequency profiling», en Proceedings of the Workshop on Comparing Corpora Volume 9. WCC'00. Stroudsbourg, PA, USA: Association for Computational Linguistics: 1-6. http://dx.doi.org/10.3115/1117729.1117730.

Scott, M. y Tribble, C. (2006): Textual Patterns: Key Words and Corpus Analysis in Language Education. Amsterdam: John Benjamins.

Sáez BAEzA, C. (2009): «Invisibilización de la comunicación alternativa: propuestas de entrada y salida», Revista Latina de Comunicación Social, 64: 416-423. http://dx.doi.org/10.4185/RLCS-64-2009-833-416-423. 
SÁnCheZ-Saus LaSerna, M. (2017): «Marcadores lingüísticos en comunicación para el desarrollo y el cambio social: principales categorías temáticas en la producción académica sobre el tema», Commons. Revista de Comunicación y Ciudadanía Digital, 6(2): 78-98. http://dx.doi.org/10.25267/ COMMONS.2017.v6.i2.05.

Servaes, J. (2003): Approaches to Development Communication, París: UneSCo.

- (2012): «Comunicación para el desarrollo y el cambio social. Una visión general», CIC. Cuadernos de Información y Comunicación, 17: 17-40. http:// dx.doi.org/10.5209/rev_CIYC.2012.v17.39256.

Sierra Caballero, F. (2000): «Comunicación educativa y economía política: apuntes sobre políticas culturales e innovación tecnológica», Revista Latina de Comunicación Social, 26. Recuperado de: https://goo.gl/vCxcvb.

Travesedo de Castilla, C. (2013): «Nuevas tecnologías para otras estrategias de comunicación para el cambio social. Más allá de las radios comunitarias», Estudios sobre el Mensaje Periodístico, 19(1): 295-309. http://dx.doi. org/10.5209/rev_ESMP.2013.v19.n1.42523.

TuFTE, T. (2006): «SIDA, globalización y seguridad ontológica», Comunicar, 26: 27-34. Recuperado de: https://goo.gl/L3fRhE.

WiLkins, K. (2000): Redeveloping Communication for Social Change: Theory, Practice \& Power. Boulder, CO: Rowman \& Littlefield Publishers, Inc. 